\section{Development of a Range of Polyploid Lines in Petunia hybrida and the Relationship of Ploidy with the Single-/ Double-flower Trait}

\author{
Guo-Gui Ning, Xue-Ping Shi, Hui-Rong Hu, Yan Yan, and Man-Zhu Bao ${ }^{1}$ \\ Key Laboratory of Horticultural Plant Biology, Ministry of Education, \\ College of Horticulture and Forestry Sciences, Huazhong Agricultural \\ University, Shizishan 1, Wuhan 430070, Hubei Province, China
}

Additional index words. chromosome doubling, double flower, Petunia hybrida, ploidy level, sexual hybridization, relationships

\begin{abstract}
A set of Petunia hybrida plants encompassing a range of ploidy levels was developed through colchicine-mediated induction of chromosome doubling. The resulting double-flower tetraploid plants were cross-hybridized with inbred single-flower diploid lines to generate $F 1$ populations with segregation for ploidy level and flower type. The initial in vivo application of colchicine to seedling apical tips produced mixoploid plants of petunia at a high rate of efficiency. Thus, $95 \%$ of the shoot tips treated with colchicine for $48 \mathrm{~h}$ resulted in polyploid mutant plants, and no difference in this efficiency was observed using concentrations of colchicine between 0.2 and $2.0 \mathrm{mg} \cdot \mathrm{mL}^{-1}$. Of the polyploid plants, $10 \%$ were found to be tetraploid and $85 \%$ were mixoploid (chimeric). Compared with their diploid counterparts, polyploid plants underwent reduced elongation growth during the first 2 weeks and had thicker stems and shorter internodes resulting in dwarfing of the whole plant. In extreme cases, very slow growth rates produced stunted plantlets. Polyploid plants also had larger, thicker leaves and, in some cases, the leaves that developed after 1 month of growth appeared seriously malformed. Octoploid plants were also obtained and these tended to have more extreme phenotypes. Pure tetraploid plants of double-flower petunia were isolated by the in vitro culture of explants from the initial chimeric tetraploid mutants. These were crossed with three inbred single-flower diploid lines (S1, S2, and S3) thereby generating F1 populations that showed segregation for flower type and ploidy level and included the generation of triploid plants. In the tetraploid plants, flower diameter and the number of flower petals were not changed significantly $(P>0.05)$ compared with the original diploid doubleflower plants, but observation of the pollen grains revealed segregation for size consistent with the increased ploidy level. Analysis of the F1 progeny plants also indicated that chromosome number is not necessary but sufficient to cause the production of semidouble-flowered plants. Flower color and flower diameter were also analyzed in the F1 progeny and complex patterns of inheritance were inferred. In addition to single and double flowers, semidouble-flowered plants were also suggested to be generated by the hybridization of $2 \mathrm{n}$ or $3 \mathrm{n}$ pollen from the double-flower tetraploid plants with the singleflower diploid lines.
\end{abstract}

In ornamental plants, flower architecture is an important feature and double-flowered varieties are particularly highly valued by the horticultural industry (Scovel et al., 1998). Double-flowered varieties have been characterized anatomically and genetically in several plant species, including Chamelaucium ucinatum and Nicotiana alata (McComb et al., 1996; Zainol and Stimar, 2001; Zainol et al., 1998). Petunia is a profuse flowering

\footnotetext{
Received for publication 18 Sept. 2008. Accepted for publication 3 Nov. 2008.

This research project is partially financed by Natural Science Foundation of China (30671477) and the Ministry of Agriculture of China (2003-Z36).

We thank Dr. Alex McCormac (UK) for critically editing this manuscript.

${ }^{1}$ To whom reprint requests should be addressed; e-mail baomanzhu@yahoo.com.cn.
}

annual with large, richly colored flowers and exists as a diverse array of varieties (Griesbach, 2007). As the most popular petunia varieties, double-flowered petunias had been well studied from an anatomical angle, but the genetic background of the double-flower form in petunia has not been investigated (Natarella and Sink, 1971; Sink, 1973; Vanderkrol and Chua,1993). Certainly, there have also been no studies in petunia regarding the relationship of ploidy with flower form (Meyerowitz et al., 1989). Currently, breeding for new varieties of double-flowered petunia is many single-flower and double-flower cultivars rarely produce double-flowered progeny. Furthermore, few lines reliably produce $100 \%$ double flowers and, in general, vegetative propagation is the most successful method of multiplying double-flowered plants of Petunia. Also, semidouble flowers appear to be a impeded by the fact that crosses between rarity within Petunia hybrida varieties. Hence, the genetic basis of the double-flower phenotype in petunia is poorly understood and this is a problem for breeding programs aimed at this important characteristic.

Polyploidy is a prominent and significant force in plant evolution (Adams and Wendel, 2005) and has played a major role in the evolution of flowering plants (Masterson, 1994; Otto and Whitton, 2000). The manipulation of ploidy can also be a valuable tool for plant breeding programs. Polyploids often generate variants that may contain useful characteristics and provide a wider germplasm base for breeding studies (Ramanna and Jacobsen, 2003; Thao et al., 2003). Since the discovery by Blakeslee and Avery (1937) of the effectiveness of colchicine for the achievement of chromosome doubling in plants, colchicine has been successfully used to obtain polyploid plants of many agricultural and ornamental crops, e.g., Phlox subulata L. (Zhang et al., 2008), Zizyphus jujuba Mill. 'Zhanhua' (Gu et al., 2005), hop (Roy et al., 2001), and bread wheat (Soriano et al., 2007). Similarly, Power and Sink (1978) produced triploid and tetraploid lines in petunia and documented the relationship of ploidy with various phenotypic characters, but because the study was conducted with single-flowered lines only, this did not provide information regarding the double-flower trait.

Kermani et al. (2003) conducted a study in Rosa, which indicated that chromosome doubling was positively reflected in the extent of flower complexity. However, there is otherwise little in the literature regarding the relationship between ploidy level and petaline type as relating to single- or doubleflower architecture.

In this article, we describe the creation of a ploidy series based on double-flowered lines of Petunia hybrida. The various ploidy levels were generated through the colchicinemediated induction of polyploid plants and cross-hybridization between double-flower tetraploid and single-flower diploid plants. We describe the major phenotypic characteristics among the various polyploid lines, including flower form. From this analysis, we note that an unusually high frequency of semidouble-flowered plants was generated from crosses involving a polyploid doubleflower plant and a diploid single-flower Petunia cultivar and investigate the relationship between ploidy level and petaline type.

\section{Materials and Methods}

Plant materials. The three single-flower lines used in this study were S1 (single whiteflowered), S2 (single brick red-flowered), and S3 (purple with white edge-flowered). These were inbred lines obtained by continuous pedigree selection during growth in the experimental field of Huazhong Agricultural University, Wuhan City, China. The seeds of double-flower petunia varieties (petunia hybrida 'double \& Valentine') were purchased from Hongyue Ltd., Hangzhou, 
China. All growth, cross-hybridization, and colchicine treatment of plants were conducted under the standard greenhouse or field conditions supplied at the field site of Huazhong Agricultural University.

Chromosome doubling by colchicine treatment. Seedlings to be treated with colchicine were obtained from diploid doubleflower petunia seed grown in a medium of peat:perlite $(3: 1)$ until the cotyledons had expanded. Strips of degreasing cotton were saturated with a solution of colchicine $(0.2$, $0.5,1.0$, or $2.0 \mathrm{mg} \cdot \mathrm{mL}^{-1}$ ) and these were wrapped around the seedling apical tips. The treated seedlings were maintained under greenhouse conditions for $48 \mathrm{~h}$, during which time the colchicine solution was reapplied to the degreasing cotton every 6 h. Finally, the degreasing cotton was removed, the tips were washed with tap water, and the treated plants were further nursed under greenhouse conditions. Phenotypic analysis of the treated plants was carried out as an initial screen to indicate a change in plant ploidy levels. This analysis included measurement of the internodal distance on the main stem, stem diameter, leaf width, leaf shape index, leaf thickness, and visual character of the leaf.

Ploidy level determination. In an initial screen for putative tetraploids, leaf samples were taken from the colchicine-treated seedlings and from control (untreated) seedlings, sampling at approximately the same point on each plant. A thin layer was removed from the abaxial surface of the leaves and analyzed for stomatal size and density under a light microscope.

For direct analysis of ploidy levels, nuclei were extracted from leaf samples and stained using the reagent kit Partec CyStain ultraviolet precise P (Partec, Münster, Germany). Young leaves were crushed slightly using a sharp razor blade in HR-A nuclei extraction buffer and, after filtration though a Partec 30 $\mu \mathrm{m}$ Cell-Trics disposable filter, HR-B staining solution was added. The samples were analyzed immediately using a PA-I Xow cytometer (Partec) equipped with a $100-\mathrm{W}$ high-pressure mercury arc lamp. A leaf from a diploid plant of Petunia hybrida was used as a control sample.

Chromosome counts were also performed on the putative polyploid plants. Actively growing apical and axillary shoot tips were excised from young plants and pretreated with saturated $\mathrm{p}$-dichlorobenzene solution for $6 \mathrm{~h}$. They were then washed with distilled water three times and fixed in ethanol-acetic acid $(3: 1)$ for $24 \mathrm{~h}$ at room temperature. The fixed shoot tips were hydrolyzed in 0.2 $\mathrm{mol} \cdot \mathrm{L}^{-1} \mathrm{HCl}$ for $15 \mathrm{~min}$ at $60^{\circ} \mathrm{C}$, after which the shoot tips were rinsed three times with distilled water and macerated in acetic acid $(45 \%)$ for 5 to $10 \mathrm{~min}$. The macerated shoot tips were placed on a microscope slide and stained with Carbol fuchsin. The preparation was gently squashed beneath a coverslip and chromosomes in metaphase spreads were counted under a light microscope.

Chimeric plant culture. The polyploid plants developing from the original colchi- cine-treated seedlings were chimeric for ploidy levels. To obtain pure tetraploid plants, tetraploid shoots or leaves were excised from the chimeras and cultured in vitro. Excised shoots were proliferated on a Murashige and Skoog (MS) medium supplemented with either $0.5 \mathrm{mg} \cdot \mathrm{L}^{-1}$ benzyladenine (BA) and $0.05 \mathrm{mg} \cdot \mathrm{L}^{-1}$ 1-Naphtaleneacetic (NAA) or $0.1 \mathrm{mg} \cdot \mathrm{L}^{-1} \mathrm{BA}$ and $0.01 \mathrm{mg} \cdot \mathrm{L}^{-1}$ NAA. Leaf explants were incubated on an MS medium containing $1.0 \mathrm{mg} \cdot \mathrm{L}^{-1} \mathrm{BA}$ and $0.05 \mathrm{mg} \cdot \mathrm{L}^{-1} \mathrm{NAA}$ to induce plantlet regeneration. The regenerated shoots were rooted on half-strength MS medium. Plantlets were acclimatized, transferred to a peat:perlite (3:1) medium, and grown in a greenhouse.

Crosspollination of double-flower tetraploids with single-flower diploid petunia lines. The pure tetraploid plants were allowed to flower and pollen samples were analyzed. The diameter of the pollen grains was measured to support the diagnosis of the parent plant's ploidy level. Pollen grains were also stained with Carbol fuchsin and observed under a light microscope to control for "giant" pollen grains. The tetraploid plants were used to pollinate three inbred singleflower lines, S1, S2, and S3. The resulting seeds were collected and grown to produce mature plants of $\mathrm{F} 1$ progeny populations.

Relationship of flower phenotype with ploidy level. The progeny plants obtained from the cross of the tetraploid plants with the inbred lines were analyzed with respect to ploidy levels according to the techniques described previously. These progeny plants were also analyzed with respect to flower characteristics such as form, diameter, and color.

Statistical analysis. Data were evaluated by analysis of variance and means were compared using Duncan's multiple range tests to determine significant differences $(P<$ $0.05)$.

\section{Results}

Colchicine treatment of seedlings and ploidy determination of plants. Seedlings of diploid double-flowered lines of Petunia hybrida were treated with various concentrations of a colchicine solution (0.2 to 2.0 $\left.\mathrm{mg} \cdot \mathrm{mL}^{-1}\right)$, which was applied to their apical tips over a period of $48 \mathrm{~h}$. The majority of seedlings survived the treatment and after 1 month, it was evident that $95 \%$ of the seedlings had developed abnormal morphologies. Based on these observations, it appeared that the different concentrations of colchicine were all equally effective for the induction of mutation $(96.4 \%, 96.5 \%, 98.0 \%$, and $100 \%$ corresponded to a solution of $0.2,0.5$, 1.0 , or $2.0 \mathrm{mg} \cdot \mathrm{mL}^{-1}$ colchicine).

The colchicine-treated seedlings showed stunted growth (Fig. 1, B1, C1) when compared with control plants (Fig. 1, A1); for example, the internodal distance on the main stem was reduced and stem diameter was increased. After 2 weeks of growth, the treated seedlings had produced only two to three true leaves compared with on these control plants, and these began to appear thicker, greener, occasionally indented, and with shorter petioles (Table 1). One month later, more than $90 \%$ of these abnormal seedlings still showed morphological abnormalities, notably larger and thinner leaves, thicker stems, shorter internodes and reduced overall plant height, increasingly evident plant hairs and, in some instances, poor growth vigor, and seriously malformed leaf growth (Fig. 1, A1-C1).

Subsequently, analysis of stomata size, cytometric analysis, and chromosome counting were carried out to determine the ploidy levels of the colchicine-derived seedlings.

Stomata size and the density of their distribution over the abaxial leaf surface were found to differ significantly $(P \leq 0.05)$ between known diploid plants and the mutated seedlings (Table 1). Thus, the stomata of most mutant plants were significantly larger than those of diploid plants, whereas the number of stomata over the leaf surface was significantly reduced (Fig. 1, A2, B2, C2).

Flow cytometric analysis was used to analyze the nuclear content of the plants, which were colchicine -treated, altered morphology, and larger stomata, and in most cases, the data histograms derived from the HR-B-stained nuclei showed one dominant peak aligning to the ploidy level of the plant. Thus, the G1 peak of nuclear preparations from expected tetraploid material aligned most strongly on channel 100 (Fig. 1, B4), whereas control plants (diploid material) showed a G1 peak on channel 50 (Fig. 1, A4). In addition, mixoploid plants with both diploid and tetraploid cells were identified by the presence of two peaks of similar height corresponding in fluorescence intensity to the G1 peaks for diploid and tetraploid plants, respectively (Fig. 1, C4). These plants were considered most likely to be chimeras.

Cytological analysis identified the chromosome number of diploid plants of Petunia hybrida as $2 \mathrm{n}=2 \mathrm{x}=14$ (Fig. 1, A3). In two of the tetraploid plants (as identified by cytometric analysis), the cells were found to be larger than those of diploid plants with 28 chromosomes in each cell, i.e., $2 n=4 x=28$ (Fig. 1, B3). As well as other tetraploid individuals, some plants were found to be octoploids or chimeras (Fig. 1, C3). Cytological analysis was carried out on 20 individuals selected as putative polyploids on the basis of morphological observation and stomatal analysis. The results showed that four of these plants were tetraploids, five were chimeras with both diploid and tetraploid cells, nine were chimeras with tetraploid and octoploid cells, and two were octoploids. Cells with different chromosome number were found in the same individual plant, which indicated that chimeras were also produced by the treatment of colchicine. Of 20 mutant plants tested, cytometric analysis and chromosome counting produced the same diagnosis of ploidy level in each case.

Isolation of pure tetraploid plants from chimaeras. To isolate pure tetraploid lines, two independent plants were chosen that had 

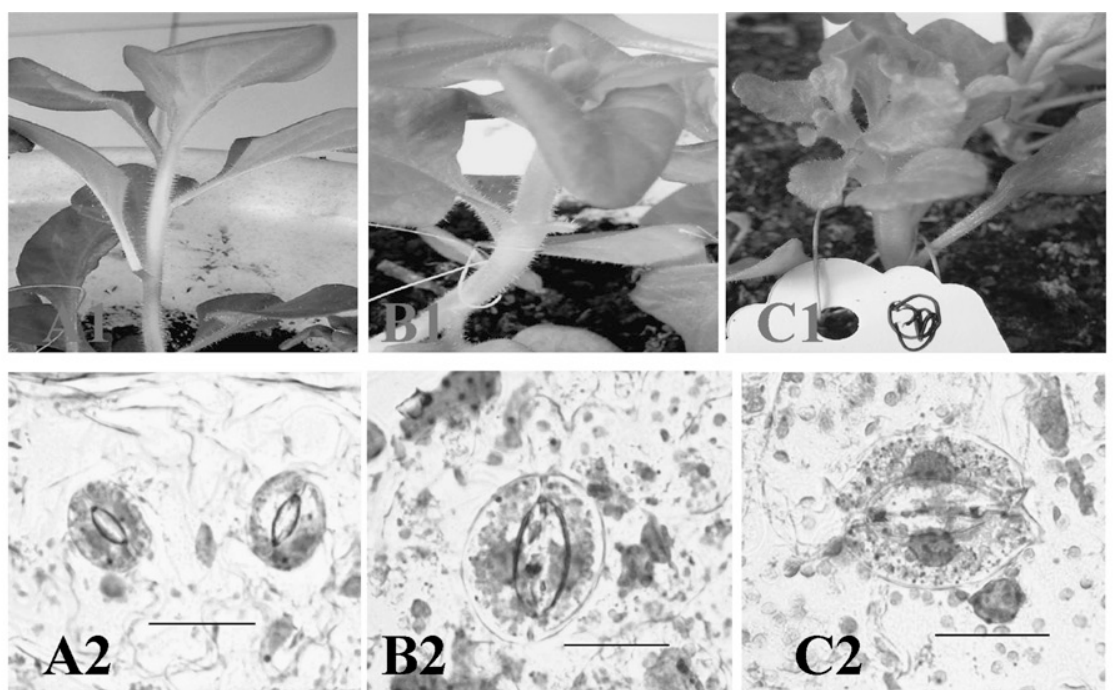

A2
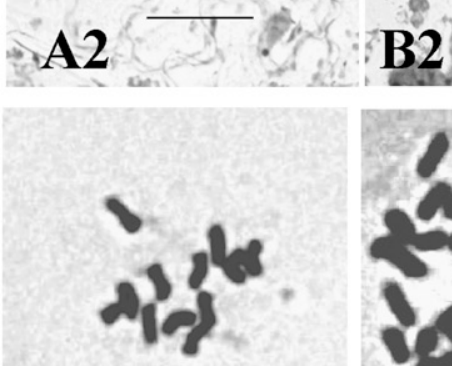

A3
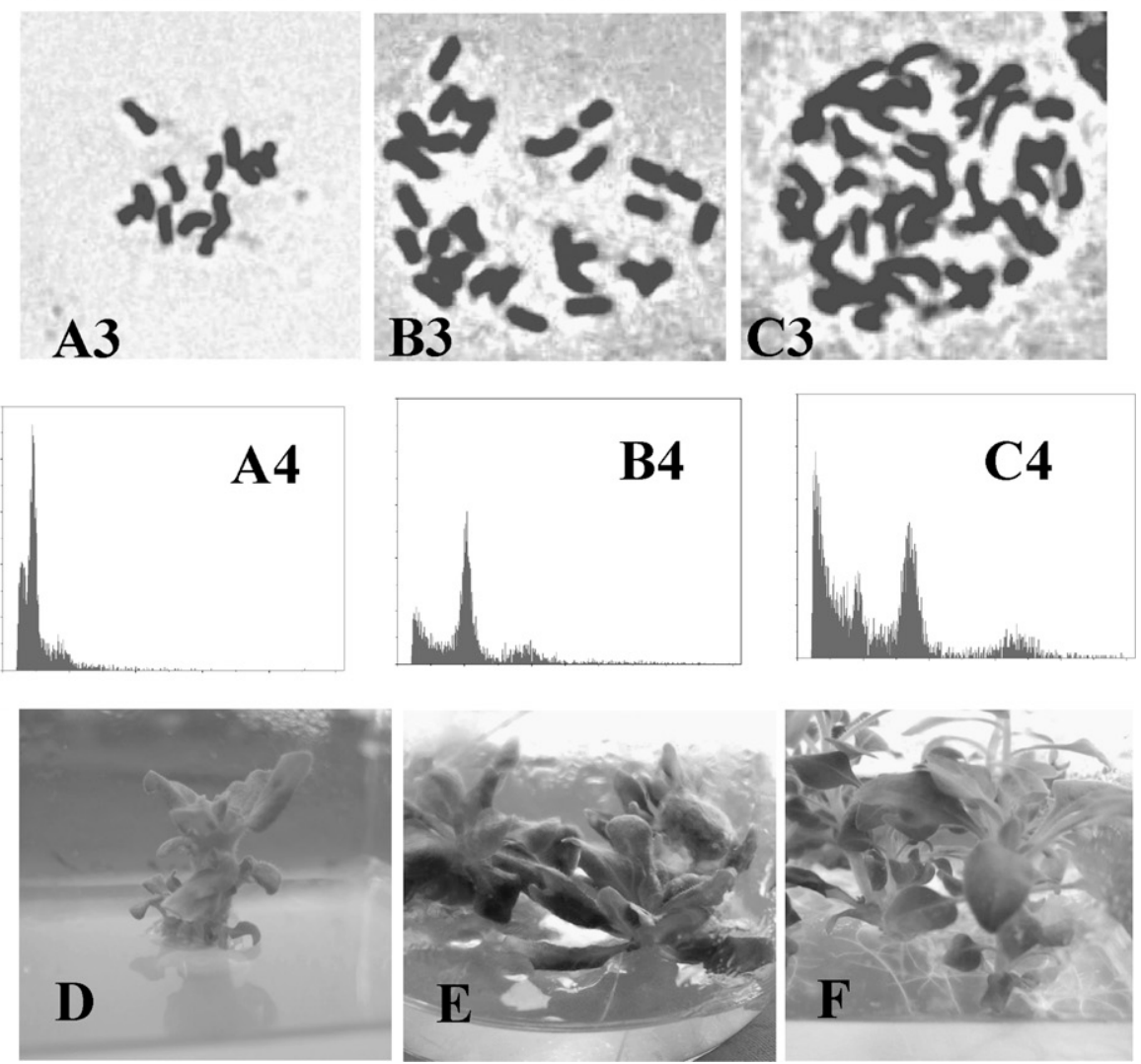

Fig. 1. Development of polyploid plants from double-flower petunia lines. (A1-A4) Plant morphological characters, stomata size, chromosome number, and flow cytometry results from young diploid plants of double-flower Petunia hybrida (the origin of the tetraploid and multiploid plants). (B1-B4) Results as stated previously, for tetraploids plants generated through colchicine application. (C1-C4) Results as stated previously, for multiploid plants generated through colchicine application. (D) In vitro culture of the stem explants from chimeric tetraploid and multiploid plants. (E) Shoot proliferation of in vitro cultures. (F) Rooting of the regenerated shoots for homogeneous tetraploid and multiploid plantlets.

developed from the apical tips of colchicinetreated seedlings and had been confirmed as tetraploid by both cytometric analysis and chromosome counting. Shoots and leaves were excised from these two plants and cultured on regeneration medium. The resulting shoots were incubated on rooting medium to obtain whole plantlets of purely tetraploid cells (Fig. 1D-F). The tetraploid plants were acclimatized and transferred to the field to mature and flower. Observation of pollen samples under a light microscope revealed differently sized pollen grains in each individual plant (Fig. 2A), suggesting that ploidy segregation had occurred. Viable seed was obtained by crossing these tetraploid plants with three inbred diploid lines.

Vegetative phenotypes effects of polyploidy. Plant morphological characteristics were compared between lines with different ploidy levels and a number of traits were identified, which showed significant differences. Com- pared with the original diploid line, the colchicine-induced tetraploid plants of petunia had significantly shorter and thicker stems (Table 1), the leaf width was increased significantly from $2.1 \mathrm{~cm}$ to $2.8 \mathrm{~cm}$, and the leaf shape index (leaf width/leaf length) was increased significantly from 0.74 (diploid, control) to 0.84 (tetraploid) (Table 1). The leaves were also thicker in the tetraploid plants, thereby appearing greener (Table 1). Also in multiploid plants (i.e., $2 \mathrm{n}>2 \mathrm{x}$ ), the internodal distance on the main stem was significantly decreased and the stem diameter increased compared with control (diploid) plants (Table 1). Furthermore, the internodal distance was significantly shorter than that even of the tetraploid plants. However, the leaf width and leaf index values were not significantly different from control plants (Table 1) and thus these quantitative traits in multiploid plants were significantly smaller than in the tetraploids. The thickness and color of the leaves of multiploid plants was comparable to those in the tetraploids, being thicker and greener than in the diploids (Fig. 2D). However, leaves of multiploid $(>4 n)$ plants had the further feature of being indented along the edge rather than smooth as in tetraploid and diploid plants (Fig. 2E-F). The number of petals and the diameter of the flowers of tetraploid plants were not significantly different when compared with the original diploid plants (Fig. 3B-C). Multiploid $(>4 n)$ plants produced some flower buds, but most of them were malformed and failed to develop as fertile flowers, although those tetraploids and triploid plants did not show the same results.

Segregation of flower form with ploidy level in F1 progeny. Two homogeneous tetraploid lines were derived from the original chimeric tetraploid plants through tissue culture methods. These were used as the pollen donors for crosses with three inbred diploid single-flower lines. In the F1 progeny of each cross, both double-flower and singleflower plants were obtained, but in all F1 progeny plants with double flowers, the petal number was reduced significantly compared with their double-flowered parent. Those double-flowered F1 progeny plants, achieved from whichever inbred diploid single-flower lines were used as parents, presented change of ploidy levels, including $2 n, 3 n$, or $4 n$, and they all produced normal flower. In each case, flower color reflected the characteristics of both parents (Fig. 3A), the F1 progeny plants, either single- or double-flower plants, show the middle color being close to the two parents, including flower speckle such as obtained from the S3 single-flower line, the color of those semidouble flower plants presented white flower speckles similar to the single-flower line parents. Inheritance of the quantitative trait of flower diameter appeared more complex. The diameter of flowers in the double-flower progeny of S1 $x$ $4 \mathrm{X}$ and $\mathrm{S} 2 \times 4 \mathrm{X}$ was 7.9 and 7.3 , respectively, and this was not significantly different from that in the double-flowered parent. Flower diameter in the single-flowered progeny of 
Table 1. Comparison of plant morphological characters in double-flower Petunia hybrida plants with various ploidy levels.

\begin{tabular}{|c|c|c|c|c|}
\hline & Diploids & Tetraploids & Multiploid & Triploid $^{z}$ \\
\hline Internodal distance of main stem $(\mathrm{cm})$ & $3.63 \pm 1.08 \mathrm{a}$ & $2.50 \pm 0.72 b$ & $1.48 \pm 0.17 \mathrm{c}$ & $2.85 \pm 0.31 \mathrm{ab}$ \\
\hline Stem diameter $(\mathrm{cm})$ & $0.49 \pm 0.05 \mathrm{~b}$ & $0.68 \pm 0.11 \mathrm{a}$ & $0.64 \pm 0-06 \mathrm{a}$ & $0.69 \pm 0.07 \mathrm{a}$ \\
\hline Breadth of leaf $(\mathrm{cm})$ & $2.1 \pm 0.22 \mathrm{~b}$ & $2.8 \pm 0.25 \mathrm{a}$ & $2.1 \pm 0.26 \mathrm{~b}$ & $2.3 \pm 0.19 \mathrm{~b}$ \\
\hline Leaf index (width/length) & $0.74 \pm 0.06 \mathrm{bc}$ & $0.84 \pm 0.07 \mathrm{a}$ & $0.70 \pm 0.06 \mathrm{c}$ & $0.80 \pm 0.04 \mathrm{ab}$ \\
\hline Thickness of leaf (mm) & $0.48 \pm 0.05 \mathrm{~b}$ & $0.64 \pm 0.08 \mathrm{a}$ & $0.68 \pm 0.04 \mathrm{a}$ & $0.5 \pm 0.02 \mathrm{~b}$ \\
\hline Visual character of leaf & Light green, smooth & Green, smooth & Green, accidented & Light green, smooth \\
\hline Length of stomata (um) & $13.6 \pm 2.95 \mathrm{c}$ & $19.7 \pm 3.51 \mathrm{~b}$ & $30.8 \pm 2.07 \mathrm{a}$ & $15.7 \pm 2.00 \mathrm{bc}$ \\
\hline Width of stomata (um) & $11.3 \pm 2.18 b$ & $14.1 \pm 1.94 \mathrm{~b}$ & $22.1 \pm 1.65 \mathrm{a}$ & $12.1 \pm 2.59 \mathrm{~b}$ \\
\hline Number of stomata per $\left(10 \mathrm{um}^{2}\right)$ & $7.2 \pm 2.22 \mathrm{a}$ & $2.7 \pm 1.71 \mathrm{bc}$ & $1.0 \pm 0.82 \mathrm{c}$ & $4.0 \pm 1.82 b$ \\
\hline Length of petiole $(\mathrm{cm})$ & $3.2 \pm 0.34 \mathrm{a}$ & $1.9 \pm 0.09 \mathrm{c}$ & $1.3 \pm 0.23 \mathrm{~d}$ & $2.3 \pm 0.12 \mathrm{~b}$ \\
\hline
\end{tabular}

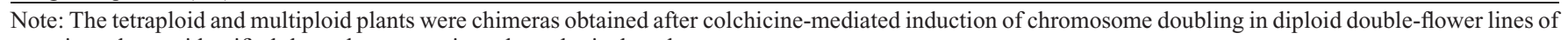
petunia and were identified through cytometric and cytological analyses.

${ }^{\mathrm{z}}$ The triploid plants were derived from a crossing of pure tetraploid plants (derived after the tissue culture of chimeric tetraploids) and the diploid inbred line S2. Each value represents the mean \pm SE of more than five plant individuals (the tetraploid plants derived from the two pured clones). Values in each row followed by different letters are significantly different $(P<0.05)$.
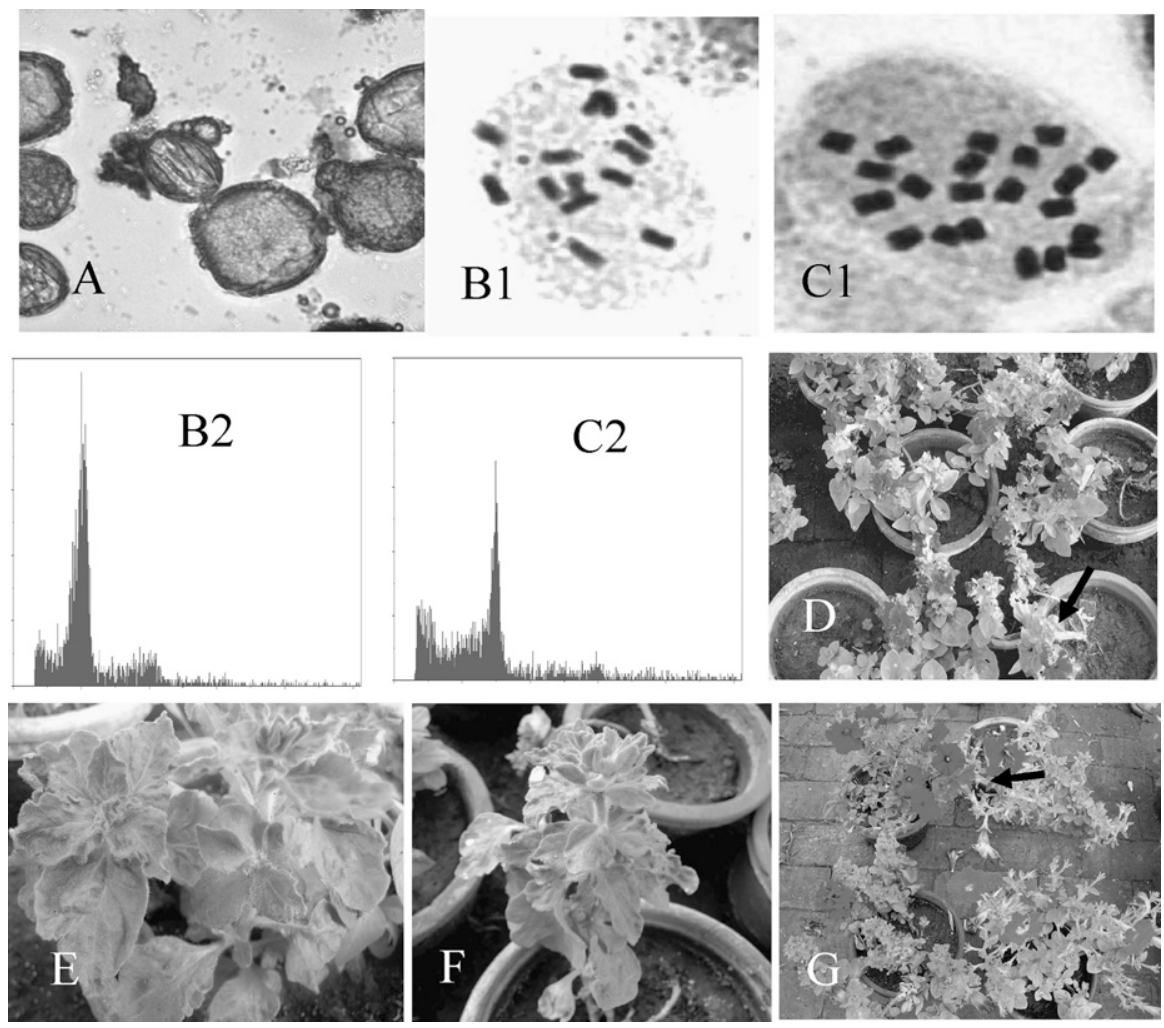

Fig. 2. Analysis of F1 progeny Petunia hybrida plants from tetraploid (double-flower) $\times$ diploid (singleflower) cross. (A) Mature pollen of tetraploid plants. (B1-B2) Chromosome number and flow cytometry results of diploid F1 plants. (C1-C2) Chromosome number and flow cytometry results of triploid F1 plants. (D) The mature flowering plants of diploids and tetraploids (arrow indicates the tetraploid plants). (E) Multiploid plants with mild phenotypic effects. (F) Multiploid plants with severe phenotypic effects. (G) Triploid F1 plants with the double-flower form (indicated by arrow) alongside the tetraploid double-flower parent, single-flower parent, and sibling plants.

these two crosses was 6.9 and 6.5 , respectively, and in the former cross, this corresponded to a significant increase compared with the S1 maternal parent (flower diameter $=5.4)$ and in the latter cross showed no difference from S2 (flower diameter $=5.8$ ) (Fig. 3C). Flower diameter in all progeny plants of the $\mathrm{S} 3 \times 4 \mathrm{X}$ cross was the same as that of the $4 \mathrm{X}$ parent and this was significantly increased compared with that of the S3 parent. Cytometric analysis and chromosome counting (Fig. 2, B1, B2, C1, C2) were carried out in 38 randomly chosen $\mathrm{F} 1$ progeny plants. The results showed that all 17 of the single-flower plants were diploid and that two semidouble-flower (showing more petals than single flower, but dramatically decreasing compared with double flower) progeny plants from $\mathrm{S} 1 \times 4 \mathrm{X}$ and $\mathrm{S} 2 \times 4 \mathrm{X}$ crosses were also diploids. Interestingly, the semidouble-flower progeny all (Fig. 2G) were polyploid and included triploids and tetraploids; they did not fit the rule that all the double or semidouble-flower F1 progeny plants were polyploid, in which diploid plants were involved, and that otherwise all the semidouble-flowered plants were determined as polyploid.

\section{Discussion}

We report the efficient in vivo induction of chromosome doubling in double-flower varieties of $P$. hybrida by the treatment of seedling shoot tips with colchicine. We describe the morphological characteristics of the polyploids and the relationship of ploidy level with the double-flower trait and flower diameter. This provides some of the first information regarding the relationship of ploidy levels with floral architectural traits in petunia and so has relevance to breeding strategies for the improvement of doubleflowered petunia lines.

In this study, we found no influence of the concentration of colchicine $(0.2$ to 2.0 $\mathrm{mg} \cdot \mathrm{mL}^{-1}$ ) that was applied to the apical shoot tip on the effectiveness for mutant induction in the double-flowered petunia lines. Plants showing chromosome-doubling were derived from a high proportion (95\%) of the original treated seedlings. Plants were also generated that appeared to have inconsistent numbers of chromosomes suggesting that in addition to tetraploid plants, chimeras had been produced. Chimeras have also been reported in several other species after the application of colchicine either in vitro (Shao et al., 2003; Thao et al., 2003) or in vivo (Schifino and Fernandes, 1987). Therefore, we used the in vitro culture of leaves or stems from the chimeras to regenerate homogeneous tetraploid plantlets and after rooting and acclimatization, these developed as tetraploid plants of double-flowered petunia. Power and Sink (1978) have previously reported the production of tetraploid plants of single-flower petunia through a similar route of colchicine application and in vitro culture of shoot tips regenerated from the resulting chimeras.

In several different plant species, polyploidy is known to be associated with multiple phenotypic effects, typically enlargement of the flowers, leaves, and fruits, enhanced duration of flowering, darker leaves, an increase in leaf shape index (leaf width/leaf length), and improvements in resistance to pests and tolerance to stress (Kehr, 1996; Zhang et al., 2008). We report that colchicine-induced tetraploid plants of doubleflower petunia also display these trends. Furthermore, plants with higher levels of 

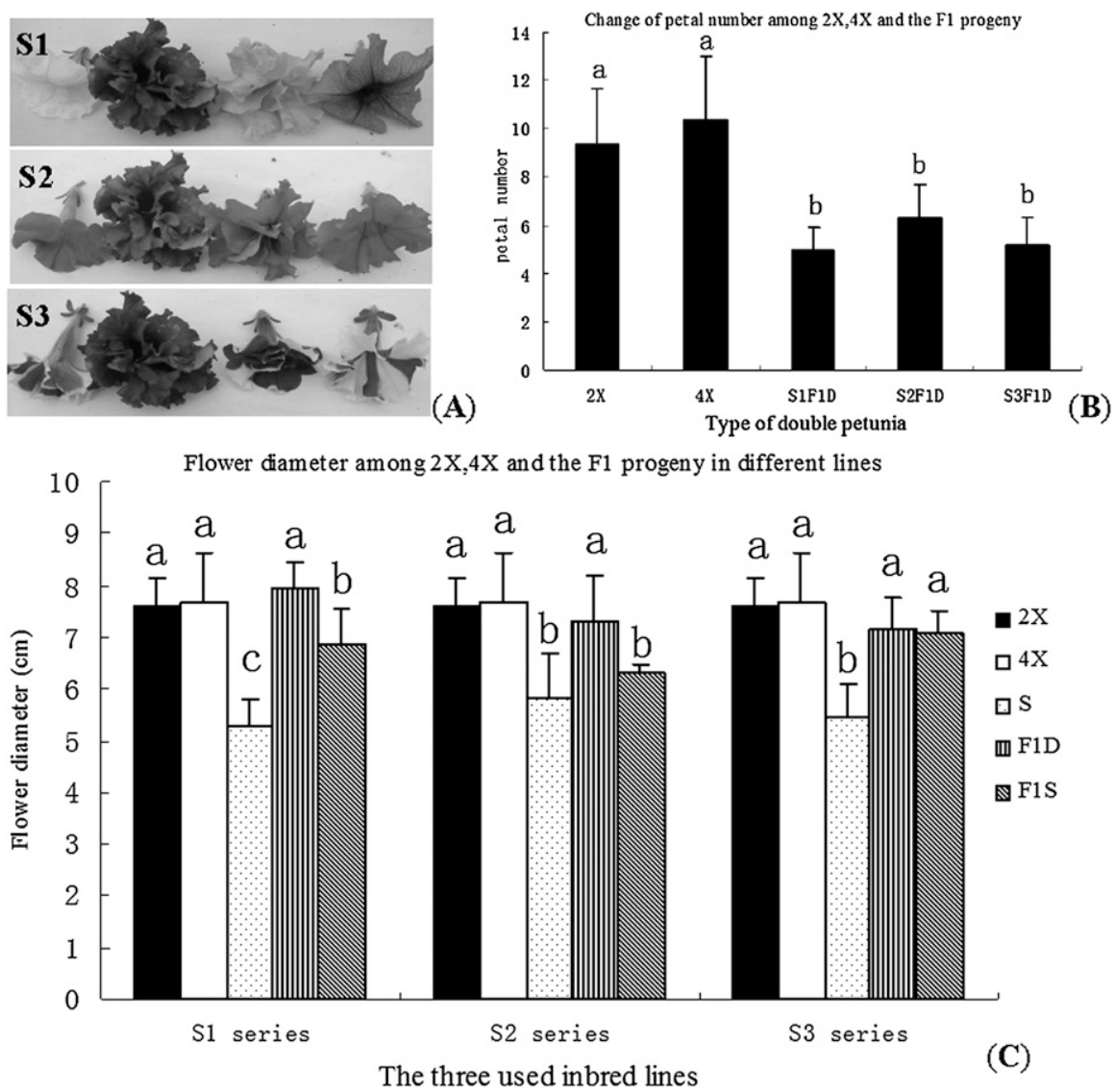

Fig. 3. Comparison of flower phenotype (flower color, petal number, and flower diameter) among diploid, tetraploid, and triploid plants of a segregating F1 population from crosses between double-flower tetraploid petunia and inbred diploid single-flower lines. (A) S1, S2, and S3 indicate the three inbred diploid single-flower parent lines used in each cross. Frames from left to right: female diploid parent; male tetraploid parent; F1 plant with double flower; F1 plants with single flower. (B) Comparison of petal number in plants that were: diploid (2X) double-flowered, tetraploid (4X) double-flowered, and double-flowered F1 progeny plants (F1D) from a cross of this tetraploid with single-flower inbred lines $\mathrm{S} 1, \mathrm{~S} 2$, or S3. (C) Comparison of flower diameter in 2X and 4X double-flower plants, $2 \mathrm{X}$ single-flower inbred line, and in the F1 progeny of the cross between the double-flower tetraploid and diploid singleflower inbred line (shown separately for the three crosses with S1, S2, and S3 female parents). Note: 2x, $4 \mathrm{x}$, and F1D (3x) indicates those plants with double flowers; S indicates female parent (2x), F1S (2x) indicates those plants with single flower. Note: In (B) and (C), values in each column followed by different letters are significantly different $(P=0.05)$.

polyploidy were also obtained and they showed an even more dramatic change in morphology as compared with the diploids (Fig. 2). Another typical characteristic of polyploids is an increase in stomata size and a concomitant decrease in their density over the leaf epidermis (Beck et al., 2003; Thomas et al., 2000). We also found in $P$. hybrida that a comparative analysis for these traits was an effective initial screen to discriminate polyploid plants from diploids.

It has been reported for some Rosa cultivars that chromosome doubling was associated with an increase in the number of petals (Kermani et al., 2003). In contrast, we found that flower diameter and the number of petals were not changed significantly in tetraploid petunia plants, whereas the double-flower trait was also retained as in the original diploid line. However, we also observed that petunia plants with higher levels of polyploidy developed as mature plants but failed to produce fertile flowers, although several abnormal flower buds were seen. Thus, it seems that floral development is in fact subject to the effects of chromosome doubling. It may be that there is a dose effect of the genes responsible for the double-flower trait that impacts on general flower development, but this suggestion will require further investigation.

The pure tetraploid double-flower plants were used as the pollen donors in crosses with three inbred diploid single-flower lines. In the F1 progeny populations, the diploid plants showed flower characteristics (e.g., flower color and diameter) that tended to reflect those of the maternal parent. However, some diploid progeny plants also displayed the double-flower trait of the male parent (two semidouble-flower progeny plants from $\mathrm{S} 1 \times$ $4 \mathrm{X}$ and $\mathrm{S} 2 \times 4 \mathrm{X}$ crosses were also determined as diploids), demonstrating that the double-/ single-flower phenotype did not consistently cosegregate with ploidy level.

Interestingly, all polyploid individuals in the F1 population (i.e., triploid or tetraploid plants resulting from the segregated $2 n$ or $3 n$ pollen grains, respectively, of the male par- ent) had semidouble flowers, i.e., the number of petals was intermediate between the double and single forms. Thus, from the doubleflower phenotype of diploid plants and the semidouble flowers of the polyploid progeny, we infer that chromosome doubling is not necessary but sufficient to cause increased petal number. We interpret this to indicate that two or more gene loci should be responsible for the double-flower phenotype because of the existence of most of diploid progeny plants with single flowers in the F1 segregated population and few of diploid double-flower plants.

In conclusion, we obtained polyploid plants of double petunia by colchicine treatment and combined this with in vitro culture and plant regeneration to provide an efficient method of obtaining pure tetraploid plants. The tetraploid plants displayed a number of morphological differences compared with the original diploid line, but these tended to be vegetative traits and the flower diameter and petal number of the resulting tetraploid doubleflower plants were not increased significantly. Significant changes, however, occurred in both the vegetative and floral traits of plants with higher ploidy levels. Sexual hybridization between a tetraploid double-flower paternal parent and diploid single-flower maternal plant resulted in progeny populations that segregated independently for flower form (i.e., single or double flower) and ploidy level. Thus, crosses produced double-flower petunia plants of different ploidy levels, indicating that there is no direct relationship between ploidy level and flower form. Because diploid, triploid, and tetraploid double flowers of petunia were found with no significant difference in petal number, we conclude that there is no gene dosage effect with regard to this trait. Furthermore, although chromosome doubling did not increase petal number, it was found that crossing double-flower tetraploid plants with single-flower lines could produce some progeny with decreased petal numbers relative to the male parent.

\section{Literature Cited}

Adams, K.L. and J.F. Wendel. 2005. Polyploidy and genome evolution in plants. Curr. Opin. Plant Biol. 8:135-141.

Beck, S.L., R.W. Dunlop, and A. Fossey. 2003. Stomatal length and frequency as a measure of ploidy level in black wattle, Acacia mearnsii (de Wild). Bot. J. Linn. Soc. 141:177-181.

Blakeslee, A.F. and A.G. Avery. 1937. Methods of inducing doubling of chromosomes in plants by treatment with colchicine. J. Hered. 28:393-411.

Griesbach, R.J. 2007. Petunia, p. 301-336. In: Anderson, N.O. (ed.). Flower breeding and genetics. Springer, New York, NY.

Gu, X.F., A.F. Yang, H. Meng, and J.R. Zhang. 2005. In vitro induction of tetraploid plants from diploid Zizyphus jujuba Mill. cv. Zhanhua. Plant Cell Rpt. 24:671-676.

Kehr, A.E. 1996. Woody plant polyploidy. Amer. Nurseryman 183:38-47.

Kermani, M.J., V. Sarasan, A.V. Roberts, K. Yokoya, J. Wentworth, and V.K. Sieber. 2003. Oryzalininduced chromosome doubling in Rosa and its 
effect on plant morphology and pollen viability. Theor. Appl. Genet. 107:1195-1200.

Masterson, J. 1994. Stomatal size in fossil plants: Evidence for polyploidy in majority of angiosperms. Science 264:421-423.

McComb, J.A., C. Newell, and G. Lullfitz. 1996. A double-flowered geraldton wax (Chamelaucium unicinatum Schauer) with an anomalous flower in the ovary. HortScience 31:283-284.

Meyerowitz, E.M., D.R. Smyth, and J.L. Bowman. 1989. Abnormal flowers and pattern formation in floral development. Development 106:209217.

Natarella, N.J. and K.C. Sink. 1971. The morphogenesis of double flowering in Petunia hybrida. J. Amer. Soc. Hort. Sci. 96:600-602.

Otto, S.P. and J. Whitton. 2000. Polyploidy incidence and evolution. Annu. Rev. Genet. 34:401-437.

Power, J.B. and K.C. Sink. 1978. Somatic and sexual hybrids of Petunia hybrida and Petunia parodii. J. Hered. 69:373-376.

Ramanna, M.S. and E. Jacobsen. 2003. Relevance of sexual polyploidization for crop improvement-A review. Euphytica 133:3-18.
Roy, A.T., G. Leggett, and A. Koutoulis. 2001. In vitro tetraploid induction and generation of tetraploids from mixoploids in hop (Humulus lupulus L.). Plant Cell Rpt. 20:489-495.

Schifino, M.T. and M.M. Fernandes. 1987. Induction of polyploidy and cytological characterization of autotetraploids of Trifolium grandense Burkart (Leguminosae). Euphytica 36:863872.

Scovel, G., M.H. Ben, M. Ovadis, H. Itzhaki, and A. Vainstein. 1998. RAPD and RFLP markers tightly linked to the locus controlling carnation (Dianthus caryophyllus) flower type. Theor. Appl. Genet. 96:117-122.

Shao, J.Z., C.L. Chen, and X.X. Deng. 2003. In vitro induction of tetraploid in pomegranate (Punica granatum). Plant Cell Tiss. Org. 75:241-246.

Sink, K.C. 1973. The inheritance of a petalous flower type in Petunia hybrida Vilm. and linkage tests with the genes. Euphytica 22:520-526.

Soriano, M., L. Cistue, M.P. Valles, and A.M. Castillo. 2007. Effects of colchicine on anther and microspore culture of bread wheat (Triti- cum aestivum L.). Plant Cell Tiss. Org. 91: 225-234.

Thao, N.P., K. Ureshino, I. Miyajima, Y. Ozaki, and H. Okubo. 2003. Induction of tetraploids in ornamental Alocasia through colchicine and oryzalin treatments. Plant Cell Tiss. Org. $72: 19-25$.

Thomas, T.D., A.K. Bhatnagar, and S.S. Bhojwani. 2000. Production of triploid plants of mulberry (Morus alba L.) by endosperm culture. Plant Cell Rpt. 19:395-399.

Vanderkrol, A. and N.H. Chua. 1993. Flower development in petunia. Plant Cell 5:11951203.

Zainol, R. and D.P. Stimar. 2001. A monogenic recessive gene, $f w$, conditions flowers doubling in Nicotiana alata. HortScience 36:128-130.

Zainol, R., D.P. Stimart, and R.F. Evert. 1998. Anatomical analysis of double flower morphogenesis in Nicotiana alata Link \& Qttq. J. Amer. Soc. Hort. Sci. 123:967-972.

Zhang, Z., H. Dai, M. Xiao, and X. Liu. 2008. In vitro induction of tetraploids in Phlox subulata L. Euphytica 159:59-65. 